\title{
A Rheodilatometer for Molten Polymers
}

\author{
I. BRUKER* and A. S. LODGE, Rheology Research Center, \\ University of Wisconsin-Madison, 1500 Johnson Drive, \\ Madison, Wisconsin 53706
}

\section{Synopsis}

A new cone-plate rheometer has been constructed in order to measure torqueinduced sample volume change $\Delta V_{\mathrm{s}}$ as well as the usually measured total thrust $F$ and torque $M$. One aim is to use $\Delta V_{\mathrm{s}}$ data to assess the accuracy of time-dependent values $N_{1}(t)$ of the first normal stress difference calculated from $F$ data obtained in step shear and step shear rate experiments. The cone-plate gap has an $8^{\circ}$ angle and $25.4 \mathrm{~mm}$ radius. The total axial stiffness is $31.4 \mathrm{MN} / \mathrm{m}$ (when $F$ is $100 \mathrm{~N}$ ); $F$ resolution is about $1 \mathrm{~N} . \Delta V_{\mathrm{s}}$ resolution is about $0.001 \%$ at $130^{\circ} \mathrm{C}$; a silicone liquid is used as a dilatometer indicator. First applications made with a low-density polyethylene sample ("IUPAC A") at $130^{\circ} \mathrm{C}$ subjected to a step shear rate of $1 \mathrm{~s}^{-1}$ for a duration of $14 \mathrm{~s}$ gave no detectable change in sample volume; it follows that, under these conditions, the maximum possible error (caused by sample volume changes) in $N_{1}$ values is only $1.3 \%$ in our Rheodilatometer and $4.2 \%$ in the BASF Weissenberg Rheogoniometer which was modified by Meissner. It also follows that the effects of any temperature rise (e.g., due to work done in shearing the sample) are negligible in the present measurements. This gives reason to question the reliability of certain torque data recently obtained by Y.-H. Lin with a Rheometries System Four rheometer.

\section{INTRODUCTION}

The importance of being able to obtain reliable values for $N_{1}$, the first normal stress difference generated by polymeric liquids in unidirectional shear flow, is well known. Of particular importance for molten polymers are the time-dependent values generated by step shear strain or by step shear rate tests: the former, for example, when combined with shear stress data, can be used to eliminate infinite classes of otherwise $a$ priori admissible constitutive equations, ${ }^{1}$ while the latter (i.e., the values of $N_{1}(t)$ fol-

${ }^{*}$ Now with General Electric Co., Corporate R \& D, P.O. Box 8, Schenectady, NY 12345.

(c) 1985 by The Society of Rheology, Inc. Published by John Wiley \& Sons, Inc. Journal of Rheology, 29(5), 557-577 (1985) CCC 0148-6055/85/050557-21\$04.00 
lowing a step shear rate change) can give significant information about molecular structure (the pronounced maximum observed with polystyrene, ${ }^{2}$ for example, is inconsistent with the prediction of an equation of Doi and Edwards ${ }^{3}$ according to which the function $N_{1}(t)$ is monotonic increasing, even when polydispersity is taken into account). Time-dependent values of $N_{1}$ (rather than steady state values) are also of importance because in most, if not all, situations in polymer melt processing, steady state values are never attained because residence times are usually small compared with the long relaxation times possessed by commercial polymers. There is evidence ${ }^{4}$ that $N_{1}(t)$ maximum values, particularly at low shear rates, could be used to give improved quality control for certain film-blowing resins where conventional methods (intrinsic viscosity and gel permeation chromatography measurements) could not.

The great value of such $N_{1}(t)$ data for polymer melts is, unfortunately, matched by the great difficulty involved in obtaining reliable data. ${ }^{1}$ In principle, the cone-plate rotational rheometer is an absolute instrument with which $N_{1}$ may be determined from measured values of axial thrust $F$ by means of the well-known equation

$$
N_{1}=2 F /\left(\pi R^{2}\right),
$$

where $R$ denotes the radius of the sample in the cone-plate gap. In practice, however, the measurement of total thrust on the cone or plate can only be made if this thrust causes a small axial displacement $\Delta X$, say, in the cone or plate which responds elastically to the thrust. It is also possible to calculate $F$ and $N_{1}$ from measurements of the pressure distribution over the plate (instead of measuring a plate displacement $\Delta X$ ) but even in this case there will always be cone and plate displacements resulting in a coneplate gap change in response to the polymer-generated thrust $F$ because the apparatus axial stiffness $K$ must in practice be bounded and not infinitely great. The resulting cone-plate gap changes, though very small, can still give significant systematic errors, particularly in time-dependent values for $N_{1}$ for liquids (such as many polymer melts) of sufficiently high viscosity; it is not so much a question of the error involved in calculating shear rate when the gap is changing (this error is usually negligible) 
but rather a question of the dynamics of the measuring system and test sumple combined.

The small gap change generates an unwanted "squeeze flow" in the sample under test; this usually gives a negligible disturbance to the desired unidirectional shear flow but can give a significant contribution to the thrust $F$. This unwanted contribution can be made negligible by increasing the apparatus axial stiffness $K$, by using a small enough sample radius $R$, and particularly by using a large enough gap angle (in the range $6^{\circ}$ to $10^{\circ}$, for example). ${ }^{5}$

Unfortunately, the larger the stiffness $K$, the greater is the apparatus sensitivity to another possible source of systematic error, namely, volume changes induced by torque in the sample under test. Such volume changes are to be expected due to temperature rise caused by the work done on the sample: a rough adiabatic estimate gives $1.5^{\circ} \mathrm{C}$ as an upper limit on this temperature rise for LDPE in our rheodilatometer for a shear strain of 20 shear units; such a temperature rise would give an increase in volume of $0.1 \%$ which would give unacceptably large errors in $N_{1}$, if it actually occurred. Another possible source of torque-induced volume change could conceivably arise from morphological changes, in block copolymers, for example; for a styrene-butadiene-styrene block copolymer subjected to a step shear rate of 0.01 $\mathrm{s}^{-1}$ at $150^{\circ} \mathrm{C}$ in a stiffened Weissenberg Rheogoniometer (WRG), a negative axial thrust was observed, with a minimum at 20 shear units; ${ }^{2}$ if this were due to a volume decrease, a volume decrease of only $0.001 \%$ would suffice to account for the small negative thrust observed. We do not claim that a volume decrease is the true explanation in this case, because the SBS sample was found to exhibit a yield shear stress, and it is well known that the presence of a yield stress can give anomalous thrust data, ${ }^{6}$ including negative values. We do claim, however, that, in order to interpret time-dependent thrust data obtained with typical polymer melts in cone-plate rheometers which have an axial stiffness large enough to make squeeze flow errors negligible, it is essential to have information about the magnitude of torque-induced volume change in the test sample. Such information is not available in the literature, to the best of our knowledge.

A torque-induced volume change may be expected to cause a change $\Delta h$ in the cone-plate separation $h$ (evaluated at the outer 
rim, say) together with a radial velocity field in the sample. A complete calculation of these effects (necessary for an exact error analysis) would be difficult. For the present purpose, it is sufficient to note that, for a given value of $\Delta V_{\mathrm{S}}$, the greatest possible value of $|\Delta h|$, namely $\left|\Delta V_{\mathrm{S}}\right| /\left(\pi R^{2}\right)$, would be attained if the radial velocity field effects were negligible; using also the equations $\Delta F$ $=K \Delta h, V_{\mathrm{S}}=(2 \pi / 3) R^{3} \tan \alpha$, and (1), we can obtain the following inequality giving an upper limit on the error $\Delta N_{1}$ due to an ignored volume change $\Delta V_{\mathrm{S}}$ :

$$
\left|\Delta N_{1} / N_{1}\right| \leqslant\left(4 K / 3 \pi R N_{1}\right)\left|\Delta V_{\mathrm{S}} / V_{\mathrm{S}}\right| \tan \alpha,
$$

where $\alpha$ denotes the gap angle. It is evident that, as expected, the error increases with increase in stiffness $K$; squeeze flow errors, on the other hand, ${ }^{5}$ are inversely proportional to $K$.

The fundamental question therefore arises in cone-plate rheometry for any given polymer melt: can one, by making an acceptable choice of values for $K, R$, and $\alpha$, find an "experimental window" through which values for $N_{1}(t)$ of acceptable accuracy can be detected, in spite of the difficulties created by squeeze flow damping and torque-induced volume change?

In order to answer this question, we have constructed a new apparatus which is designed to supplement conventional measurements of thrust $F$ and torque $M$ generated by a polymer melt sample in a cone-plate gap with direct measurements of concomitant sample volume changes. The present paper gives a brief description of this apparatus together with a few results of initial tests. A more detailed account has been given elsewhere. ${ }^{7}$

It is perhaps instructive to use (2) to compare maximum errors to be expected from some cone-plate rheometers. We consider a case in which the maximum value of $N_{1}$ is $42 \mathrm{kPa}$ (as measured, for example, for IUPAC A LDPE at $1 \mathrm{~s}^{-1}$ and $150^{\circ} \mathrm{C}$ ), and we take $R=25.4 \mathrm{~mm}, \alpha=8^{\circ}$. If $\left|\Delta V_{\mathrm{S}} / V_{\mathrm{S}}\right|=10^{-4}$, then the corresponding upper bounds on the error $\left|\Delta N_{1}\right|$ for appropriate values of $K$ for different apparatus are found to be as follows: Gleissle ${ }^{8}(K=150$ $\mathrm{MN} / \mathrm{m} ; N_{1}$ error less than $84 \%$ ); Meissner $^{5}$ (50 MN/m; 28\%); Rheodilatometer $(31 ; 18 \%)$. A more useful comparison would include errors due to squeeze flow damping, which have recently been accurately estimated ${ }^{7}$ we plan to include such a comparison, and to include additional apparatus, in a future publication 
which will describe results of multi-step shear strain experiments performed with the Rheodilatometer.

It should be noted that, for molten polymers for which the stress and refractive index tensors are linearly related, birefringence measurements have been used to give data equivalent to $N_{1}(t)$ with reasonable agreement ${ }^{9}$ with values of $N_{1}(t)$ calculated from $F(t)$ data on the assumption that the effects of torqueinduced volume changes are negligible. Since birefringence measurements should not be significantly affected by small volume changes, such agreement strongly suggests that the effects of volume changes were negligible for the polymers measured. In the case of LDPE, this result is consistent with the negligible value of $\Delta V_{\mathrm{S}}$ measured in the Rheodilatometer (see below). In view of this result, one might ask whether Rheodilatometry is really necessary, and whether birefringence could not be used to give equivalent information instead, at least where values of $N_{1}(t)$ and shear stress are concerned. The fact is, however, that birefringence measurements are not easy either; there is no suitable birefringence instrument at present commercially available, whereas there are several rheogoniometers available and in use; and finally there are materials of interest for which birefringence measurements are impracticable: filled polymers and, perhaps, some block copolymers. For these reasons, we consider that Rheodilatometry is essential at the present time.

\begin{abstract}
APPARATUS
A schematic diagram of the Rheodilatometer is given in Figure 1. A polymer sample, preformed in the shape of the cone-plate gap by means of a vacuum compression mold, is inserted between the plate (upper) and cone (lower). The plate is attached to the upper part of the outer case by means of a "wheel spring" system having three radial fin "spokes" which deflect slightly under the influence of the axial thrust $F$ and torque $M$ which the polymer sample exerts on the plate when the cone shaft is rotated during an experiment. The vertical and angular deflections are measured by capacitance transducers (not shown) whose readings, after calibration, yield the required values of $F(t))$ and $M(t)$. The transducers are located inside the external housing and are, ac-
\end{abstract}




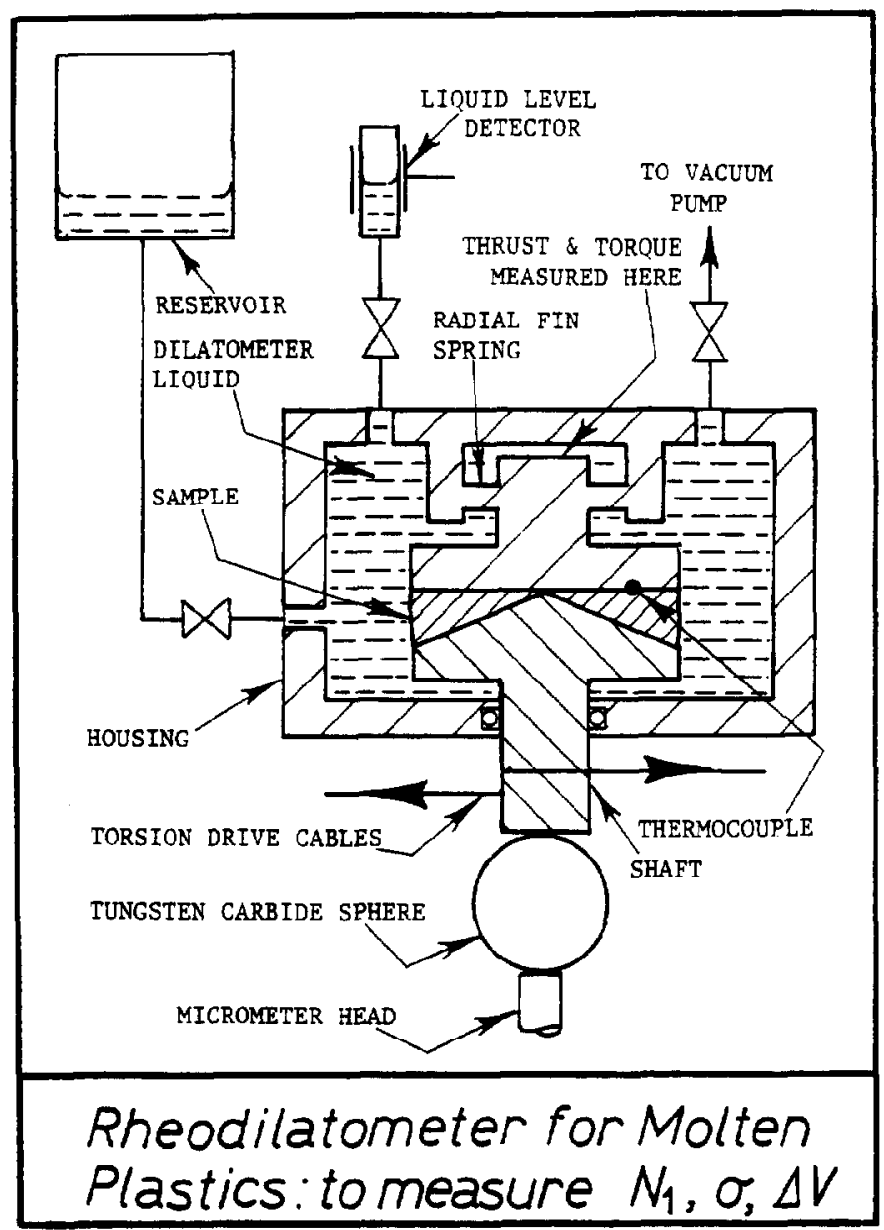

Fig. 1. Rheodilatometer schematic diagram, central section. The cone, rotated by a 4-cable system, rests on a sphere on a micrometer head. The plate is part of a 3 -spoke wheel spring system used with two capacitance transducers inside the housing to measure thrust and torque. The housing is filled with a dilatometer liquid whose level is measured by a capacitance transducer. External heaters are not shown. 
cordingly, totally immersed in the dilatometer fluid (added later). The wheel spring and plate system are machined from a single piece of tool steel, in order to avoid hysteresis which might occur if separate parts, clamped together, were used.

The cone shaft rotates in a plain journal bearing located in the outer housing; an O-ring seal is used to prevent leakage of dilatometer fluid. The lower end of the cone shaft (outside the external housing) rests on a 1-in-diam. precision-ground tungsten carbide sphere which in turn rests on the horizontal end of a micrometer spindle mounted vertically. The design is intended to give as small an axial movement of the cone shaft during rotation as possible, and also to enable one (in the absence of a polymer test sample) to calibrate the dilatometer by using the micrometer to move the cone up or down by a known amount. The cone is rotated by means of a system of four horizontal cables attached to the cone shaft and to a motor (not shown). This is intended to exert a pure torque on the cone shaft with negligible horizontal or vertical force components.

After inserting the premolded polymer sample at room temperature, the interior of the apparatus is evacuated. Then the temperature is raised (by means of external electric heaters, not shown) to the desired working temperature; the valve to the vacuum pump is closed, and the valve to the dilatometer fluid reservoir is opened to allow dilatometer fluid to fill the interior of the apparatus. It is hoped that this procedure effectively eliminates air bubbles from the system. The dilatometer fluid and polymer sample are degassed in a vacuum oven prior to use.

As a result of prior absorption tests ${ }^{7}$ for various types of silicone fluid and polyethylene, one type (SF-96-100, a dimethylpolysiloxane from the General Electric Co.) was selected for use as a dilatometer fluid for use with polyethylene. The dilatometer liquid level detector consists of a concentric cylinder electrode pair. The dilatometer liquid moves in the annulus between these electrodes and gives rise to a change in the capacitance of the system by an amount which depends on the difference in dielectric constants of air and of silicone fluid. It was found that this system, coupled with a suitable oscillator and capacitance bridge, gave sufficient sensitivity and stability for our purposes and was preferable to the alternative method involving the use of mercury as an indicating liquid. The resolution of the liquid level detector 
("LLD") is about $5 \mathrm{nl}$. The LLD is at room temperature and atmospheric pressure. There is a provision for applying known pressures to the top of the LLD in order to check whether air bubbles have been eliminated from the whole system: the known compressibility and volume of the silicone fluid in the system enable one to predict the response which would be obtained in the absence of air bubbles. ${ }^{7}$

Embedded in the surface of the plate is a fine wire thermocouple junction in contact with the polymer test sample and surrounded by a thermal insulator in order that the thermocouple output will reflect the temperature of the polymer rather than that of the plate. Temperature changes induced by torque applied to the polymer sample were found in this way to be remarkably small-less than $0.06^{\circ} \mathrm{C}$-for IUPAC A at $4 \mathrm{~s}^{-1}$ and $130^{\circ} \mathrm{C}$. The small value of this temperature rise is consistent with the small value of sample volume change measured (see below). The plate thermocouple is also used to measure the temperature at which the sample test is performed. In this connection, it should be noted that, due to heat conduction along the cone shaft, the cone temperature is expected to be a little lower than the plate temperature: calculation indicated that the difference should not exceed $0.3^{\circ} \mathrm{C}$ when the apparatus (and plate) temperature is $150^{\circ} \mathrm{C}$.

A computer system is used to control each experiment, to acquire, and to analyze data. In typical step shear strain experiments, one data point set is taken every $31 \mathrm{~ms}$. The drive system limits the maximum shear strain to about 30 shear units (when the $8^{\circ}$ gap is used): here, the strain rise time is about $0.22 \mathrm{~s}$, and there is a small strain overshoot; at a maximum shear strain of 15 shear units, the rise time is $0.12 \mathrm{~s}$ and there is no detectable overshoot.

In order to set the correct cone-plate gap, the cone is raised by means of the micrometer until the cone truncation touches the plate. This contact is indicated by a decrease in electrical resistance (the metallic housing circuit contains an insulating shim, not shown in Figure 1). The cone is then lowered by an amount equal to the previously measured cone truncation (about $25 \mu \mathrm{m}$ ).

\section{APPARATUS TESTS}

We first compare the values of thrust $F(t)$ obtained using our Rheodilatometer with the corresponding values obtained by 
Meissner ${ }^{4}$ who used a stiffened Weissenberg Rheogoniometer. We use samples of the same batch of a low-density polyethylene"IUPAC A" in step shear rate experiments at the same temperature $\left(150^{\circ} \mathrm{C}\right)$ and the same values $\left(1\right.$ and $\left.10 \mathrm{~s}^{-1}\right)$ of step shear rate. Sample dimensions are similar in both experiments: $8^{\circ}$ angle; radii $12 \mathrm{~mm}$ (Meissner), 15 and $25.4 \mathrm{~mm}$ (Rheodilatometer). In this comparison test, we did not use a dilatometer fluid. Axial stiffness values are similar: $K=50 \mathrm{MN} / \mathrm{m}$ (Meissner), $31 \mathrm{MN} / \mathrm{m}$ (Rheodilatometer). From the close agreement between $F(t)$ data obtained using gap angles of 6,8 , and $10^{\circ}$, Meissner concluded that the effects of squeeze flow damping (for a different LDPE sample) were negligible and that the value of $K$ chosen was therefore appropriate for polymer melts in this range.

At $10 \mathrm{~s}^{-1}$, our value ${ }^{7}$ for the maximum in $F(t)$, obtained with a $15-\mathrm{mm}$ radius sample, lies within the $4 \%$ scatter band obtained by Meissner in replicate tests. This agreement is good. At $1 \mathrm{~s}^{-1}$, our value ${ }^{7}$ for a $15-\mathrm{mm}$ radius sample is about $4 \%$ higher than our value for a $25.4-\mathrm{mm}$ sample and lies within the $23 \%$ scatter band obtained by Meissner in replicate tests; however, both our values lie near the low end (instead of near the middle) of Meissner's scatter band obtained in replicate tests. If this fact is significant (and we would perhaps need to perform replicate tests to find out), the trend is in the direction to be expected from the fact that our axial stiffness is lower than Meissner's, because this should mean that squeeze flow damping (if significant) would tend to reduce our thrust maxima in comparison with Meissner's.

From the extent of the agreement between our results and Meissner's, coupled with the extensive tests for squeeze flow damping performed by Meissner with different gap angles, we conclude that our Rheodilatometer is giving reliable thrust data (at least for LDPE) and that errors due to squeeze flow damping are acceptably small. A more detailed quantitative estimate of squeeze flow damping errors has been made on the basis of Wagner's constitutive equation. For another LDPE sample (IUPAC C) at $150^{\circ} \mathrm{C}$ and $2 \mathrm{~s}^{-1}$, the calculated squeeze flow damping contribution $^{7}$ to the $F(t)$ maximum leads to a difference of only $2 \%$ when compared with data obtained in our laboratories using another stiffened Weissenberg Rheogoniometer $\left(K=11.3 \mathrm{MN} / \mathrm{m} ; \alpha=8^{\circ}\right.$; $R=12 \mathrm{~mm})^{2}$

We conclude that, under the above conditions of test, all three 
instruments (two stiffened Weissenberg Rheogoniometers and one Rheodilatometer) give thrust data in step shear rate tests that are substantially unaffected by squeeze flow damping errors. We may therefore turn to the question: are they also unaffected by torque-induced sample volume changes?

The sample volume $V_{\mathrm{S}}=4.82 \mathrm{ml}$ when the sample fills the cone-plate gap (i.e., when the sample radius is $25.4 \mathrm{ml}$ ). In order to measure a $0.001 \%$ change in sample volume, we must resolve a change $\Delta V_{\mathrm{S}}=48 \mathrm{nl}$ in the presence of other possibly larger unwanted contributions to the dilatometer liquid level indicator signal $\Delta V_{L}$, say. There are two systematic sources of such unwanted contributions: the vertical displacement of the cone shaft (of radius $a$, say) through a distance $\Delta X_{\mathrm{L}}$, say, leading to a volume displacement $\Delta V_{\mathrm{C}}$ of dilatometer liquid given by the equation

$$
\Delta V_{\mathrm{C}}=\pi a^{2} \Delta X_{\mathrm{L}}
$$

and a change $\Delta T$ in temperature of the tool steel apparatus outer enclosure and its dilatometer liquid contents, leading to a volume displacement $\Delta V_{\mathrm{T}}$ of dilatometer liquid given by the equation

$$
\Delta V_{\mathrm{T}}=\beta V_{\mathrm{L}} \Delta T,
$$

where $V_{\mathrm{L}}$ denotes the volume of dilatometer liquid and $\beta$ its thermal expansion coefficient relative to that of the steel enclosure. Fortunately, the various volume change contributions should be additive and mutually independent, and hence we may safely assume that

$$
\Delta V_{\mathrm{S}}=\Delta V_{\mathrm{L}}-\Delta V_{\mathrm{C}}-\Delta V_{\mathrm{T}} .
$$

Since the polymer sample exerts the same thrust $F$ on the cone and on the plate, we may calculate the value of $\Delta V_{\mathrm{C}}$ from the measured value of $\Delta X_{U}$, the displacement of the upper plate (i.e., the plate) by means of the equation

$$
\Delta V_{\mathrm{C}}=\pi a^{2}\left(K_{\mathrm{U}} / K_{\mathrm{L}}\right) \Delta X_{\mathrm{U}}
$$

where $K_{\mathrm{U}}$ and $K_{\mathrm{L}}$ denote the values of stiffness for the "upper system" (comprising the plate and wheel spring unit) and the "lower system" (comprising the cone, cone shaft, tungsten carbide sphere, micrometer and micrometer support), respectively. In separate measurements made with suitable weights when the upper system was removed, the values $K_{\mathrm{U}}=145 \mathrm{MN} / \mathrm{m}$ and $K_{\mathrm{L}}=$ 
$40 \mathrm{MN} / \mathrm{m}$ (at $10 \mathrm{~kg}$ ) were obtained, giving a total stiffness $K=$ $\left(K_{\mathrm{U}}{ }^{-1}+K_{\mathrm{L}}^{-1}\right)^{-1}=31 \mathrm{MN} / \mathrm{m}$. The shaft radius $a=6.35 \mathrm{~mm}$.

The stiffness $K_{\mathrm{L}}$ is actually a function of load, due to the nonlinear relation between load and displacement at the "Hertz" contact region between sphere and flat. This nonlinearity is most pronounced at low loads-in order to make the nonlinearity insignificant in use, the shaft was preloaded (by means of a Belleville-type disc spring, not shown in Figure 1, attached to the shaft). In preliminary loading experiments, ${ }^{7}$ the force-displacement relation was carefully studied, with agreement between measured and calculated stiffnesses. In using (6) to determine $\Delta V_{\mathrm{C}}$ for correcting dilatometer measurements in a complete experiment (e.g., as in Figure 4, lowest part), a single value for $K_{\mathrm{L}}$ was used, instead of a function $K_{\mathrm{L}}(F)$ which would, in principle, have been a more accurate usage; according to our estimates, however, the error involved in using a single value of $K_{\mathrm{L}}$ was only a few percent, and thus was negligible in relation tc the scatter of the $\Delta V_{\mathrm{S}}$ data.

Equation (6) is used to evaluate $\Delta V_{\mathrm{C}}$ (for substitution in (5)) when the apparatus is used for polymer sample measurement under an applied torque. As a check on (6), and as a partial test of the proper functioning of the dilatometer system, we used the micrometer to move the cone through a known vertical distance $\Delta X_{\mathrm{L}}$ when the system was filled with dilatometer liquid with no polymer sample in the cone-plate gap; chart recordings of the measured value of $\Delta V_{L}$ versus time are shown in Figure 2 (lower part); the upper part gives directly the shaft displacement, measured by means of another capacitance transducer attached to the cone shaft (not shown in Figure 1) so as to measure capacitance to the underside of the enclosure wall. It is seen from Figure 2 that the liquid transducer signal $\left(\propto \Delta V_{L}\right)$ in the absence of shaft movement is a straight line inclined to the time axis; this inclination is attributed to a time-dependent varying-temperature contribution $\Delta V_{\mathrm{T}}$, which is about $70 \%$ greater than that calculated from direct temperature measurements, ${ }^{7}$ the reason for this discrepancy is not known; it should not affect the accuracy of our data.

By turning the micrometer twice, we obtain three "base lines" displaced from each other. When such "base lines" are parallel, they may be used to make the correction for temperature changing at a constant rate; on taking the difference in height between 


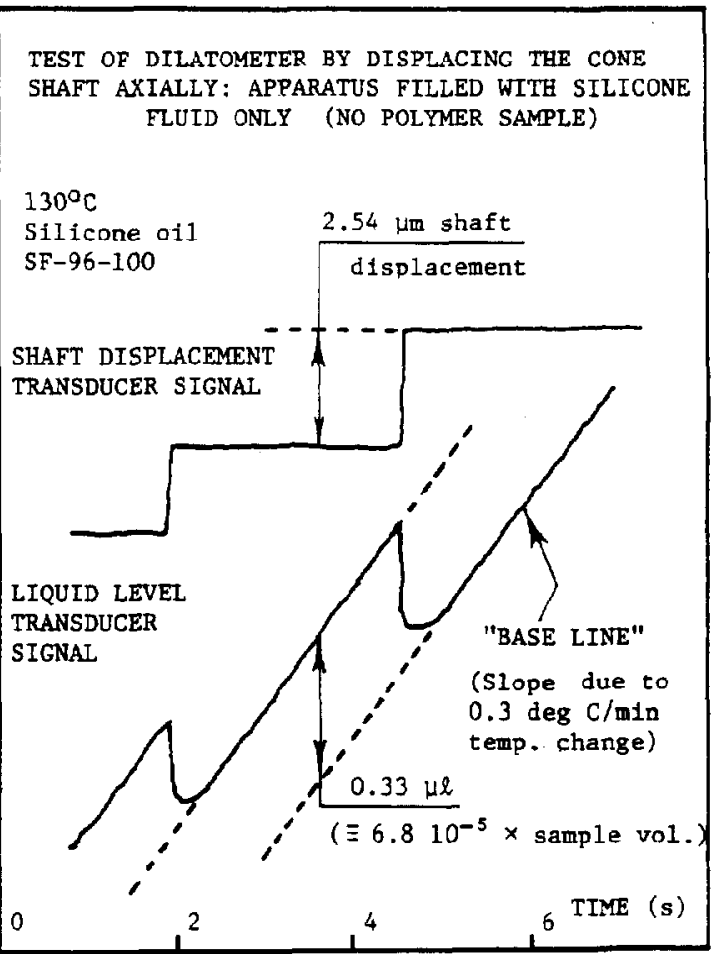

Fig. 2. Test of the Rheodilatometer without a polymer sample. The base line slope is attributed to a constant rate of change of temperature of housing and dilatometer liquid. The change in liquid level relative to this base line is caused by upward movement of the cone and shaft.

the base lines in Figure 2, we obtain a value $\Delta V_{\mathrm{C}}=0.33 \mu \mathrm{l}$ (as measured by the liquid level detector; this compares with a value of $0.32 \mu \mathrm{l}$ obtained from (3) with $\Delta X_{\mathrm{L}}=2.54 \mu \mathrm{m}$. The liquid level detector is calibrated in a separate experiment by displacing a known small volume of liquid (measured with a microsyringe) through the level detector. The above agreement gives confidence in the validity of our method of making corrections for $\Delta V_{C}$ and $\Delta V_{\mathbf{T}}$.

Figure 3 shows results of a further test made by rotating the cone, again in the presence of dilatometer liquid without a poly- 


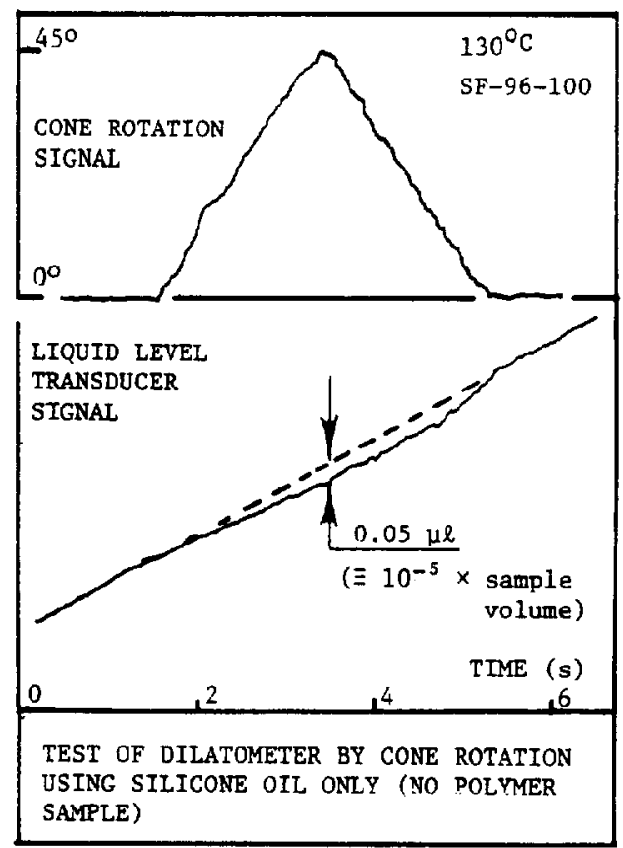

Fig. 3. Test of the Rheodilatometer without a polymer sample. The cone is rotated through $45^{\circ}$ at constant angular speed first in one direction and then in the other (upper trace). The lower trace shows a small change in the liquid level detector signal (relative to the base line); this change represents the resolution of the dilatometer.

mer sample. The upper trace* represents cone angular position versus time. The cone was rotated steadily through $45^{\circ}$ and then rotated steadily in the opposite direction through $45^{\circ}$. If the apparatus worked perfectly, the cone rotation should cause no change in the dilatometer reading. In fact, as seen from Figure 3, there was a small change in liquid level (represented by the displacement of the lower chart record below its straight base line). The reason for this is not known, but it is possibly due to a displacement of the O-ring located on the journal bearing: it is un-

* The undue noise in this trace is due to the fact that, for this test, the axial displacement was used to give a rough measurement of cone angular position. 
likely that all the air on the lower side of this O-ring could be removed by the evacuation of the apparatus, and so there would be room for a small displacement of the O-ring during cone rotation. Whatever the correct explanation may be, it is seen that the unwanted contribution is small (equal to about $10^{-5} V_{\mathrm{S}}$ ). This is of the order of the resolution of the whole dilatometer/apparatus system.

When the lower structure stiffness was measured, it was found that loading and unloading the cone resulted in some hysteresis in the cone shaft displacement signal ${ }^{7}$; this, too, might be due to friction exerted by the O-ring.

\section{POLYETHYLENE DATA}

Figure 4 shows chart recordings of $N_{1}$ (upper curve) and dilatometer liquid level indicator reading $V_{\mathrm{L}}$ (middle curve) obtained when a low-density polyethylene sample (IUPAC " $\mathrm{A}$ ") is subjected to a step shear rate of $1 \mathrm{~s}^{-1}$ at $130^{\circ} \mathrm{C}$. The horizontal axes are common time axes. After about $14 \mathrm{~s}$ (i.e., a total strain of 14 shear units), the cone rotation is stopped and stress relaxation occurs. Shear stress was not measured because, due to a design error, the wheel spring torsional stiffness ( $2.85 \mathrm{mNm} /$ radian) was greater than had been intended, with the result that the torque measurement was not sufficiently sensitive in relation to the noise in the signal. (The wheel spring spokes can be modified readily to rectify this trouble without giving an unacceptable decrease in axial stiffness.)

The temperature of $130^{\circ} \mathrm{C}$ (rather than the preferred $150^{\circ} \mathrm{C}$ at which most data ${ }^{4}$ for the IUPAC samples have been taken) was chosen because it was found that the silicone liquid used for the dilatometric measurement started to foam at $150^{\circ} \mathrm{C}$. The supplier believed that this liquid should be stable up to $200^{\circ} \mathrm{C}$. The reason for foaming at $150^{\circ} \mathrm{C}$ is not known to us. It is the onset of foaming that limits the useful temperature range of the present Rheodilatometer. (IUPAC " $\mathrm{A}$ " at $150^{\circ} \mathrm{C}$ may now be regarded as a reference material which can usefully be used to test rheological instruments, although supplies are, unfortunately, very limited.)

It is seen from Figure 4 (upper curve) that the maximum value of $N_{1}(t)$ is attained after a shear strain somewhat greater than 10 


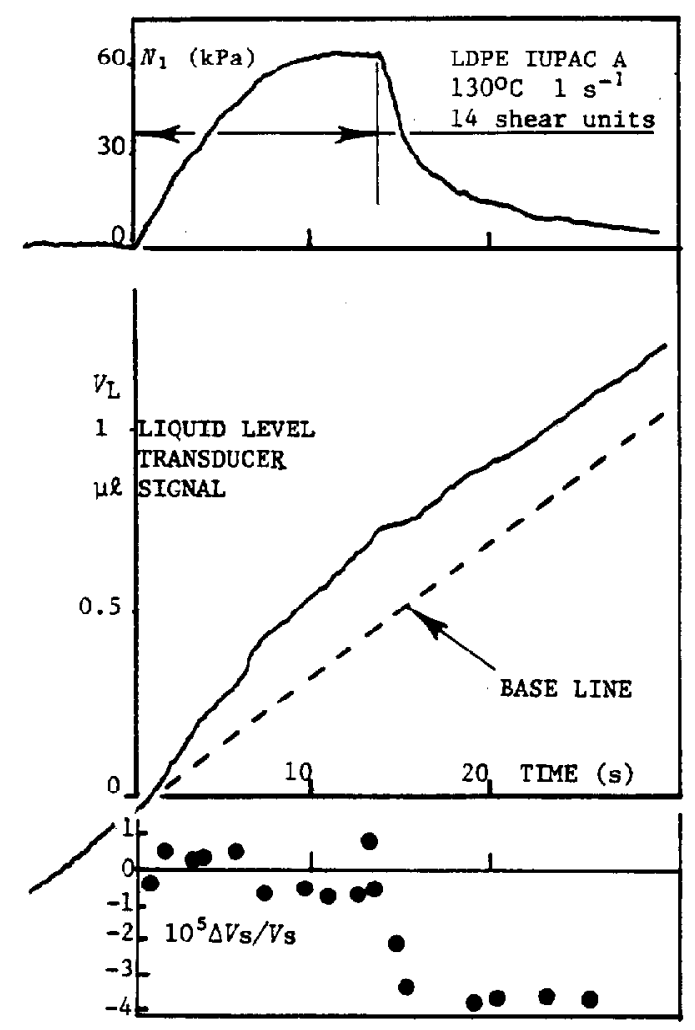

Fig. 4. Rheodilatometer data for a step shear rate of $1 \mathrm{~s}^{-1}$ for a duration of $14 \mathrm{~s}$ Top trace: first normal stress difference $N_{1}$ versus time showing stress growth and relaxation when cone rotation is stopped after $14 \mathrm{~s}$. Middle trace: liquid level transducer signal versus time on the same time axis. Lower trace: values of relative sample volume change versus time calculated from the difference between the middle trace and its base line after subtraction of the cone shaft displacement contribution $\Delta V_{\mathrm{C}}$ calculated from $\mathrm{E}_{\mathrm{Y}}$. (3) and the thrust displacement signal $\Delta X_{\mathrm{U}}$. The decrease in $\Delta V_{\mathrm{S}}$ during relaxation is an apparatus artefact due to hysteresis in the cone shaft support structure. The points between 0 and $14 \mathrm{~s}$ show no volume change within a resolution of $\pm 0.001 \%$. This is the main conclusion. 
shear units. This is consistent with data obtained by Meissner ${ }^{4}$ at $150^{\circ} \mathrm{C}$.

The dashed line in Figure 4 (middle) represents the base line (extrapolated) for the liquid level indicator; its non-zero slope is attributed to a non-zero rate of change of temperature. It is seen that, during the period in which $N_{1}$ is increasing, the liquid level indicator signal moves significantly from the base line; this movement, however, is caused by the cone shaft displacement $\Delta X_{\mathrm{L}}$ (due to axial thrust $F$ ) leading to a change $\Delta V_{C}$ in the effective volume of the apparatus; when the appropriate corrections for $\Delta V_{C}$ and $\Delta V_{\mathrm{T}}$ are made (using the base line for the latter and (6) combined with measured values of $\Delta X_{\mathrm{U}}$ for the former), the resulting values for $\Delta V_{\mathrm{S}} / V_{\mathrm{S}}$ (obtained from (5)) are seen to be scattered within the range $\pm 10^{-5}$ during the period of $N_{1}$ growth. This scatter represents the resolution of our $\Delta V_{\mathrm{S}}$ measuring technique in the present Rheodilatometer.

We conclude that, for the polymer tested at $1 \mathrm{~s}^{-1}$ and $130^{\circ} \mathrm{C}$, the torque-induced volume change does not exceed $0.001 \%$; the corresponding error in $N_{1}$ (maximum value) is less than 1.3\%. To the best of our knowledge, this is the first published result of its kind.

The above applies to stress growth. During stress relaxation, it appears from Figure 4 (lowest data points) that there is a systematic decrease in $\Delta V_{S}$; we believe, however, that this is an apparatus artifact associated with the hysteresis in the lower structure referred to above. On unloading the cone, the lower structure does not behave as a perfectly elastic structure would; the consequent error in using the "perfect elastic responses" Eq. (6) is in a direction which would account qualitatively for the decrease shown in Figure 4 during relaxation (lowest points). This hysteresis represents a limitation on the accuracy of the present apparatus when used in situations in which the thrust $F(t)$ is a decreasing function of time $t$.

We have been unsuccessful in our attempts to repeat the above experiments with the SBS sample for which Huang ${ }^{2}$ observed negative thrust values at $0.01 \mathrm{~s}^{-1}$ and $150^{\circ} \mathrm{C}$. Our present drive system prevented our going to shear rates lower than $1 \mathrm{~s}^{-1}$. In replicate tests at $1 \mathrm{~s}^{-1}$ and $130^{\circ} \mathrm{C}$, we were unable to obtain reproducible volume change data - inspection of samples after shearing showed evidence of failure of bonding between sample and platens. Such evidence was not found with the polyethylene used. 


\section{DISCUSSION}

Our main conclusion is that measured values of $N_{1}(t)$ are subject to negligible errors due to sample volume change induced by torque. This has been demonstrated for one LDPE (IUPAC $A$ at $\left.130^{\circ} \mathrm{C}\right)$ and one step shear rate $\left(1 \mathrm{~s}^{-1}\right)$ applied for a total shear strain of 14 shear units. The maximum value of this error calculated for the Rheodilatometer (using (2) with $\left|\Delta V_{\mathrm{S}} / V_{\mathrm{S}}\right| \leqslant 10^{-5}$ ) is $0.76 \mathrm{kPa}$ in $N_{1}$; this is $1.3 \%$ of the measured maximum value of $N_{1}$, namely, $60 \mathrm{kPa}$. Using the values $K=50 \mathrm{MN} / \mathrm{m}$ and $R=1.2$ $\mathrm{cm}$ appropriate to Meissner's Weissenberg Rheogoniometer, ${ }^{4}$ the same volume change would give a maximum error of $2.49 \mathrm{kPa}$ in $N_{1}$; this is greater than our error bound because the stiffness is greater and the radius smaller than ours. This error bound is $4.2 \%$ of the maximum value of $N_{1}$ and is thus comparable with the scatter usually obtained in replicate measurements. ${ }^{4}$ Squeeze flow damping errors in our Rheodilatometer would be expected to be a little larger than those in Meissner's WRG; the evidence (mentioned above) is that both are negligible.

Of particular significance are the step shear strain measurements made by Laun ${ }^{10}$ with the same WRG as that used by Meissner. Over a shear strain range of 0.2 to 30.9 shear units, Laun found that (a) $N_{1}(s, t)=s \sigma(s, t)$, where $\sigma$ denotes the shear stress and $s$ denotes the magnitude of shear strain, and $(\mathrm{b}) \sigma(s, t)=$ $s G(t) h(s)$, i.e., that the shear stress function (and hence also $N_{1}(s, t)$ ) is factorizable into a product of a function of time and a function of strain. The result (a) implies that, during relaxation after finite step shear strains, the stress and strain ellipsoids are coaxial. This is referred to as "Class I" behavior according to a recently proposed classification of constitutive equations. ${ }^{1}$ Laun's published data ${ }^{10}$ are for a LDPE "Melt 1 " at $150^{\circ} \mathrm{C}$. This LDPE is the same as another IUPAC sample-" $\mathrm{B}$ "- to which stabilizer has been added. Its rheological properties are very close to those of IUPAC A which was used in the Rheodilatometer measurements described above.

Laun analyzed his Rheogoniometer data on the assumption that errors due to torque-induced volume changes were negligible. Can we use our Rheodilatometer data to assess the validity of this assumption? It is reasonable to take the value $2.49 \mathrm{kPa}$ as an upper bound on errors in $N_{1}$ (due to ignored volume changes not 
exceeding $0.001 \%$ ) for the case of shear strains of 14 shear units: this is the error bound derived above for Meissner's WRG for different (but not very different) sample and temperature. It is also reasonable to assume that $\Delta V_{\mathrm{S}}$, and hence also the upper error bound, is proportional to the shear strain $s$-at least, for the sake of the present attempt to estimate upper bounds on error. This assumption is certainly reasonable if $\Delta V_{\mathrm{S}}$ is due to a temperature rise caused by the work done on the sample. The upper bound on the error in $N_{1}$ (due to $\Delta V_{\mathrm{S}}$ ) will then be $2.49 \mathrm{~s} / 14 \mathrm{kPa}$. Using Laun's Figure 5 data for $N_{1}$ at selected strains $s=6$ and 30.9 and selected times $t=1$ and $10 \mathrm{~s}$, we obtain the following upper bounds for the errors in $N_{1}$ due to ignored torque-induced volume changes: for $s=6: 2 \%$ at $1 \mathrm{~s}$ and $12 \%$ at $10 \mathrm{~s}$; for $s=30.9$ : $6 \%$ at $1 \mathrm{~s}$ and $38 \%$ at $10 \mathrm{~s}$. These errors are not serious in comparison with the scatter of data and with the range of variation. We conclude that torque-induced volume change errors are unlikely to have been significant, and that Laun's conclusions (a) and (b) are supported; to be more certain, however, it would be advisable to extend our $\Delta V_{\mathrm{S}}$ measurements to step strain experiments.

Laun ${ }^{11}$ has found that (a) and (b) also apply for polystyrene $\left(\mathbf{M}_{\mathbf{w}}\right.$ $\left.=240,000 ; \mathrm{M}_{\mathrm{w}} / \mathrm{M}_{\mathrm{n}}=2.76\right)$ at $170^{\circ} \mathrm{C}$; other data for the same sample have been published. ${ }^{12}$ (a) and (b) are valid over a range $0.17 \leqslant s \leqslant 35.9$. An adiabatic estimate ${ }^{11}$ for the maximum temperature rise in these experiments gave the result $\Delta T<1.64^{\circ} \mathrm{C}$. Using a volume thermal expansion coefficient value of $2.410^{-4}$ / ${ }^{\circ} \mathrm{C}$, this would give $\Delta V_{\mathrm{S}} / V_{\mathrm{S}}<4 \times 10^{-4}$, which is 40 times larger than the upper limit $10^{-5}$ obtained from Rheodilatometer measurements with LDPE. If a temperature rise of this magnitude $\left(1.6^{\circ} \mathrm{C}\right)$ actually occurred and persisted during stress relaxation, one could expect significant errors in $N_{1}$.

$\operatorname{Lin}^{13}$ has recently measured $\sigma(s, t)$ for polystyrene $\left(\mathrm{M}_{\mathrm{w}}=\right.$ 186,$000 ; \mathrm{M}_{\mathrm{w}} / \mathrm{M}_{\mathrm{n}}=1.07$ ) at $185^{\circ} \mathrm{C}$ in the range $0.2 \leqslant s \leqslant 20$. He use a Rheometrics System Four with $R=5 \mathrm{~mm}$ and $\alpha=1.03^{\circ}$. He found that $\sigma(s, t)$ was not factorizable, in conflict with Laun's results quoted above for a different polystyrene sample. Lin postulated a temperature increase of $12^{\circ} \mathrm{C}$ lasting for periods of up to $50 \mathrm{~s}$ caused by the work done in shearing the sample. This is a very serious postulate: if it is valid, it would imply that shearing the sample causes volume changes of such magnitude that would 
make it virtually impossible to calculate $N_{1}(t)$ from thrust data in cone-plate rheometers.

From the above discussion, it is clear that an error analysis for $N_{1}(t)$ measurements would be helped if there were available a calculation of time-dependent temperature fields in the coneplate system for step shear and step shear rate experiments. In the absence of such a calculation, we make two rough estimates which, perhaps, suffice for the purposes of the present discussion.

First, we can use the result $\left|\Delta V_{\mathrm{S}} / V_{\mathrm{S}}\right| \leqslant 10^{-5}$ obtained for LDPE by direct measurement in the Rheodilatometer to obtain a strict upper bound on the average temperature rise (averaged, that is, throughout the sample): using a value $9 \times 10^{-4} /{ }^{\circ} \mathrm{C}$ for the bulk expansion coefficient, we find that $\overline{\Delta T} \leqslant 0.01^{\circ} \mathrm{C}$. This is compatible with our direct measurement by means of a thermocouple in contact with the melt, which gave a temperature change of about $0.005^{\circ} \mathrm{C}$. The above volume change result refers to a step shear rate experiment at $1 \mathrm{~s}^{-1}$ with a duration of 14 shear units. For the same experiment, we obtain a calculated value of $0.21^{\circ} \mathrm{C}$ for $\Delta T_{\max }$, the maximum possible temperature rise which could occur in the sample if all the work done, namely $\int \sigma d s$ per unit volume, resulted in a temperature rise with no heat loss. This was evaluated from Meissner's data ${ }^{4}$ (Figure 16, p. 596, at $150^{\circ} \mathrm{C}$; converted to $130^{\circ} \mathrm{C}$ using the ratio of viscosities from Table 11, p. 568) together with the values $C_{\mathrm{p}}=2570$ and $\rho=769$ (all SI units). It is seen that the adiabatic estimate $\Delta T_{\max }$ overestimates the actual average temperature rise $\overline{\Delta T}$ by a factor of at least 21 -an encouraging, if somewhat surprising, result.

Secondly, we may make a very crude estimate of the effect of heat conduction to the cone and plate, assumed to be held at constant and equal temperatures. We neglect heat conduction in a radial direction-whether to the outside atmosphere or within the melt. Then, for each small cylindrical annular element of melt, the decay of an initial temperature difference is governed by the time constant ${ }^{15} \tau=\left(\rho C_{\mathrm{p}} / 4 k\right) r^{2} \tan ^{2} \alpha$, where $\rho, C_{\mathrm{p}}$, and $k$ denote density, specific heat, and thermal conductivity, and $r$ is the radius. As an extreme case, we may assume that the work done is instantaneous and produces a temperature rise that is uniform throughout the melt in the cone-plate gap. We may then use the well-known curves ${ }^{15}$ for the subsequent time variation of 
temperature appropriate to an infinite parallel-sided slab of width $2 b=r \tan \alpha$ in order to get a rough idea about temperature decay in the cone-plate system. The slowest decay will then occur midway between cone and plate at the outer $\operatorname{rim} r=R$ : the curves show that about $90 \%$ of the initial temperature difference will be lost after a time $\tau$ has elapsed. We obtain the following values for the decay time $\tau$ : for polystyrene, $8.7 \mathrm{~s}$ (Laun-Meissner WRG) and $0.023 \mathrm{~s}$ (Lin, System Four); for LDPE, $5.8 \mathrm{~s}$ (LaunMeissner WRG) and $26 \mathrm{~s}$ (Rheodilatometer).

These estimates suggest that a significant temperature decay will occur in our Rheodilatometer during the $14 \mathrm{~s}$ interval used in the $\Delta V_{\mathrm{S}}$ measurement experiment at a step shear rate of $1 \mathrm{~s}^{-1}$ (described above) and, further, that in a step shear strain experiment the temperature rise may be significantly greater than in a step shear rate experiment. It would, therefore, be advisable to measure $\Delta V_{\mathrm{S}}$ in step shear strain experiments.

The very small decay time for Lin's experiment, coupled with our upper limit on temperature rise obtained from volume change and direct temperature measurement, strongly suggest that Lin's conjectured temperature rise of $12^{\circ} \mathrm{C}$ over a $50 \mathrm{~s}$ time interval cannot have actually occurred, and hence that some other explanation for his non-factorizable torque data must be sought. A referee has suggested that, because $M_{w} / M_{n}$ is 1.07 for Lin's PS sample and is 2.8 for Laun's PS sample, factorizability for the latter need not imply factorizability for the former; the PS data of Osaki and Kurata ${ }^{14}$ (admittedly for concentrated solutions, instead of undiluted melts), however, tend to discount this suggestion, showing that factorizability occurs even up to values of $M_{w} /$ $\mathrm{M}_{\mathrm{n}}=4$, provided that $\mathrm{cM}<10^{6} \mathrm{~g} / \mathrm{ml}$ (where $\mathrm{c}=$ concentration in $\mathrm{g} / \mathrm{ml}$ ), a condition satisfied by Lin's sample.

The evidence suggests, therefore, that Lin's torque data are anomalous, as he himself believed. One possible explanation for the anomaly that should be considered (but was not considered in Lin's paper) arises from the unwanted coupling between thrust and torque signals which sometimes occurs with the Rheometrics System Four rheometer ${ }^{16}$. If, in fact, there was significant coupling between these signals in Lin's rheometer, the thrusts exerted, which would increase rapidly with increasing shear strain, would be expected to give significant torque signal errors of magnitudes which would also increase rapidly with increasing shear 
strain. It is true that Lin checked his torque measurements by comparison with some of Meissner's LDPE torque data, but if this test was made only at small strains, it would be clearly inconclusive; the strain range for this test was not stated in Lin's paper.

We are not claiming that Lin's System Four rheometer actually did give significant errors in torque measurements, because the published data of which we are aware is insufficient to substantiate (or to dismiss) such a claim. We do assert, however, that thrust-torque coupling is a possible explanation of Lin's torque data anomaly which should be considered as an alternative to Lin's proposed temperature rise explanation, which we claim is unacceptable.

For financial support, we are grateful to the National Science Foundation (Grant no. CME-7824180 to A.S.L.) and to E. I. duPont de Nemours \& Co. (for Polymer Research and Engineering Grants to the Rheology Research Center). We are grateful to $\mathrm{Mr}$ R. C. Williams for machining the apparatus, to $\mathrm{Mr} \mathrm{R}$. $\mathrm{P}$. MeCabe and Mr D. H. Hutchins for the design and construction of the electronic circuits, and to Dr Alex Stuecheli for the design and construction of a vacuum compression mold. We are indebted to a referee for helpful comments.

\section{REFERENCES}

1. A. S. Lodge, J. Non-Newtonian Fluid Mech., 14, 67 (1984).

2. T. A. Huang, Ph.D. Thesis, Univ. of Wisconsin-Madison, 1976.

3. M. Doi and S. F. Edwards, J. Chem. Soc., Faraday Trans. II, 74, 1818 (1978).

4. J. Meissner, Pure and Applied Chemistry, 42, 553 (1975).

5. J. Meissner, J. Appl. Polymer Sci., 16, 2877 (1972).

6. J. F. Hutton, Rheol. Acta, 14, 979 (1975).

7. I. Bruker, Ph.D. Thesis. Univ. of Wisconsin-Madison, 1983.

8. W. Gleissle, Ph.D. Thesis, Univ. Karlsruhe, 1978.

9. H. Janeschitz-Kriegel, Polymer Melt Rheology and Flow Birefringence, Springer, Berlin, 1983.

10. H. M. Laun, Rheol. Acta, 17, 1 (1978).

11. H. M. Laun, private communication, 1984.

12. H. M. Laun, M. H. Wagner, and H. Janeschitz-Kriegel, Rheol. Acta, 18, 615 (1979).

13. Y.-H. Lin, J. Rheol., 28(1), 1 (1984).

14. K. Osaki and M. Kurata, Macromolecules, 13, 671 (1980).

15. R. B. Bird, W. E. Stewart and E. N. Lightfoot, Transport Phenomena, 4th

Printing, Wiley, New York, 1984, p. 356, fig. 11.1-1

16. G. A. Alvarez, private communication, 1984.

Received September 11, 1984; in revised form January 18, 1985 